\title{
Optical Imaging of Renal Cell Carcinoma with Anti-Carbonic Anhydrase IX Monoclonal Antibody Girentuximab
}

\author{
Constantijn H.J. Muselaers ${ }^{1,2}$, Alexander B. Stillebroer ${ }^{1,2}$, Mark Rijpkema ${ }^{2}$, Gerben M. Franssen², Egbert Oosterwijk ${ }^{1}$, \\ Peter F.A. Mulders ${ }^{1}$, Wim J.G. Oyen ${ }^{2}$, and Otto C. Boerman ${ }^{2}$ \\ ${ }^{I}$ Department of Urology, Radboud University Medical Center, Nijmegen, The Netherlands; and ${ }^{2}$ Department of Nuclear Medicine, \\ Radboud University Medical Center, Nijmegen, The Netherlands
}

\begin{abstract}
Near-infrared dye-tagged antibodies can be used for the sensitive detection of tumor tissue in vivo. Surgery for clear-cell renal cell carcinoma (ccRCC) might benefit from the use of optical imaging to facilitate the intraoperative detection of carbonic anhydrase IX (CAIX)-expressing tumor lesions with chimeric monoclonal antibody $(\mathrm{mAb})$ girentuximab, which has been shown to have excellent imaging capabilities for ccRCC. Here we studied the potential of fluorescence imaging to detect ccRCC tumors in nude mice with RCC xenografts by using mAb girentuximab conjugated with IRDye800CW; SPECT imaging was used as a reference. Methods: Groups of athymic BALB/c mice with subcutaneous CAIX-positive SK-RC-52 ccRCC tumors were injected intravenously with ${ }^{125}$-labeled girentuximab-IRDye $800 \mathrm{CW}$ or ${ }^{125}$-labeled girentuximab. For determination of the specificity of the accumulation of the anti-CAIX antibody conjugate in cCRCC, separate groups of mice bearing a CAIX-positive tumor (SK-RC-52) and a CAIXnegative tumor (SK-RC-59) received ${ }^{125}$ I-girentuximab-IRDye800CW or ${ }^{125}$-labeled MOPC21-IRDye800CW (control mAb). Optical images and micro-SPECT images were acquired until $3 \mathrm{~d}$ after injection. Mice were euthanized after the last imaging session, and the biodistribution of the radiolabeled antibody preparations was determined. Results: Optical imaging and micro-SPECT imaging at $1 \mathrm{~d}$ after the injection of ${ }^{125}$ I-girentuximab-IRDye $800 \mathrm{CW}$ showed clear delineation of the CAIX-expressing CcRCC xenografts, and image contrast improved with time. Fluorescence imaging and biodistribution studies showed high and specific uptake of ${ }^{125}$-girentuximab-IRDye800CW in CAIXpositive ccRCC xenografts (SK-RC-52, $31.5 \pm 9.6$ percentage injected dose per gram [\%ID/g] at $72 \mathrm{~h}$ after injection). Tumor uptake was specific, as very low uptake of ${ }^{125}$-girentuximab-IRDye $800 \mathrm{CW}$ was noted in the CAIX-negative SK-RC-59 tumor $(4.1 \pm 1.5 \% \mathrm{ID} / \mathrm{g})$, and no uptake of ${ }^{125}$ I-MOPC21-IRDye800CW (control mAb) was noted in the CAIX-positive SK-RC-52 tumor (1.2 $\pm 0.1 \% \mathrm{ID} / \mathrm{g})$. Conclusion: Subcutaneous CAIX-expressing ccRCC xenografts were visualized by optical imaging with ${ }^{125}$-girentuximab-IRDye $800 \mathrm{CW}$. Optical images showed good concordance with micro-SPECT images. The accumulation of ${ }^{125}$-girentuximab-IRDye800CW in ccRCC tumors was high and specific. Girentuximab-IRDye800CW potentially could be used for the intraoperative detection of CAIX-expressing tumors and the assessment of residual tumor in resection margins or metastatic lesions in patients with $\mathrm{CCRCC}$.
\end{abstract}

\footnotetext{
Received Jan. 13, 2014; revision accepted Feb. 6, 2014.

For correspondence or reprints contact: Constantijn H.J. Muselaers, Department of Nuclear Medicine, Radboud University Medical Center, Geert Grooteplein 8, 6525 GA Nijmegen, The Netherlands.

E-mail: stijn.muselaers@radboudumc.com

Published online Apr. 21, 2014.

COPYRIGHT (C 2014 by the Society of Nuclear Medicine and Molecular Imaging, Inc.
}

Key Words: renal cell carcinoma; optical imaging; girentuximab; monoclonal antibody; CAIX

J Nucl Med 2014; 55:1035-1040

DOI: 10.2967/jnumed.114.137356

$\mathbf{R}$ enal cell carcinoma (RCC) accounts for approximately $2 \%$ of all malignancies (1). Surgical resection is usually the first choice for localized disease, but when disease has metastasized, the prognosis is bleak. Because of the increased use of conventional radiologic modalities, such as CT and ultrasound (US), RCCs are being found more frequently and at earlier stages of the disease (2). The recommended treatment for a localized RCC depends on the tumor size. Nephron-sparing surgery is performed for smaller RCCs (especially those $\leq 4 \mathrm{~cm}$ in diameter) (3) because overall survival data for T1 to T3 tumors are comparable for partial nephrectomy and radical nephrectomy (4). Negative surgical resection margins are crucial for the clinical outcome because they prevent additional surgical interventions (5). Therefore, adequate characterization and resection of the renal tumor and metastatic lymph nodes are of utmost importance.

The use of near-infrared (NIR) imaging probes for improved tumor characterization and delineation during surgery is increasing, although clinical applications have been limited. The sensitivity of NIR imaging might be extremely high, and because of the low level of tissue autofluorescence in this part of the light spectrum, the use of NIR imaging probes could yield optimal visualization of tissues of interest. Unfortunately, the limited depth of penetration of the light emitted from these agents hampers whole-body applications (6).

Fluorescent agent IRDye800CW, a typical example of an NIR dye, emits NIR 789-nm photons when excited at $774 \mathrm{~nm}$. IRDye800CW can be stably coupled to targeting molecules, such as peptides and monoclonal antibodies (mAbs), and can be detected with an NIR imaging camera $(7,8)$.

For clear-cell RCC (ccRCC) — the most common subtype of RCC, present in approximately $85 \%$ of patients with a malignant renal tumor-the chimeric mAb girentuximab (also known as cG250) could serve as a targeting molecule. Girentuximab specifically recognizes carbonic anhydrase IX (CAIX), a tumor-associated antigen ubiquitously expressed in ccRCC. The use of girentuximab in radioimmunoscintigraphy and radioimmunotherapy to detect or treat ccRCC has been investigated extensively $(9,10)$. Because of the high-level and specific targeting and accumulation of girentuximab in ccRCC tumors, we hypothesized that this antibody could 
also act as a carrier for the delivery of IRDye $800 \mathrm{CW}$ to these tumors. The intravenous administration of girentuximabIRDye800CW before (partial) nephrectomy might facilitate the sensitive, in vivo, intraoperative detection of positive resection margins, metastatic lymph nodes, or distant ccRCC metastases.

Here we characterized girentuximab-IRDye $800 \mathrm{CW}$ in a subcutaneous mouse model. The antibody-IRDye $800 \mathrm{CW}$ conjugate was radiolabeled to quantitatively determine the biodistribution. Studies were conducted to demonstrate tumor-specific and antigen-mediated uptake of the girentuximab-IRDye800CW conjugate. In addition, the tumor targeting and biodistribution of the conjugate were compared with those of unconjugated radiolabeled girentuximab. This NIR imaging system potentially could be used intraoperatively to enhance tumor visualization and resection in the near future.

\section{MATERIALS AND METHODS}

\section{ccRCC Tumors in Nude Mice}

Cell Lines. The ccRCC cell lines SK-RC-52 (CAIX-positive) and SK-RC-59 (CAIX-negative) were derived from metastases of primary ccRCC tumors as described by Ebert et al. (11). Cells were cultured and washed as described previously (12).

Animal Model. The animal experiments were approved by the Internal Review Board of Radboud University Medical Center, Nijmegen, The Netherlands, and were performed in accordance with that organization's guidelines. Animals were housed and fed according to Dutch animal welfare regulations. For obtaining subcutaneous tumors, $2 \times 10^{6}-3 \times$ $10^{6}$ cells were injected subcutaneously into the flanks of 6 - to 8 -wk-old male BALB/c nu/nu mice (Janvier); tumors grew to a size of $50-200 \mathrm{~mm}^{3}$ within 2-3 wk.

\section{Imaging Agents and Labeling}

$m A b$ Girentuximab. The isolation and immunohistochemical reactivity of mAb G250 have been described elsewhere (13). For reducing the immunogenicity of murine G250 in humans, a chimeric version (girentuximab) has been developed (14). mAb girentuximab is reactive with transmembrane glycoprotein CAIX (association constant, $4 \times 10^{9} \mathrm{M}^{-1}$ ). The expression of CAIX on the surface of ccRCC cells is ubiquitous $(>95 \%)$, whereas the expression on normal tissues is restricted to the epithelial structures of the upper gastrointestinal tract and larger bile ducts $(15,16)$.

$m A b M O P C 21$. MOPC21, a murine IgG1 mAb (Sigma-Aldrich) that is not directed against any known antigen, was used as a nonspecific control $\mathrm{mAb}$ in this study.

Conjugation, Radiolabeling, and Quality Control. Conjugation of mAb girentuximab and mAb MOPC21 with IRDye800CW (LICOR Biosciences) was performed in accordance with the supplier's protocol. One milligram of mAb was incubated with $0.03 \mathrm{mg}$ of IRDye $800 \mathrm{CW}$ for $2 \mathrm{~h}$ at room temperature in $1 \mathrm{~mL}$ of $10 \mathrm{mM}$ phosphate buffer $(\mathrm{pH}$ 8.5). The reaction mixture was transferred to a Slide-A-Lyzer cassette (molecular weight cutoff, 10,000 Da) (Pierce) and dialyzed extensively against phosphate-buffered saline for $3 \mathrm{~d}$ to remove unconjugated IRDye800CW. On average, 1.6 IRDye800CW molecules were conjugated per mAb molecule, as determined spectrophotometrically. The concentration of the conjugate was adjusted to $1 \mathrm{mg} / \mathrm{mL}$, and aliquots were stored in the dark at $-20^{\circ} \mathrm{C}$ until use. Girentuximab and girentuximab-IRDye800CW were radioiodinated with ${ }^{125} \mathrm{I}$ (PerkinElmer) by the IODO-GEN (Pierce) method (14). ${ }^{125}$ I-girentuximab was purified by gel filtration on a PD10 column (Amersham Biosciences); elution was done with phosphate-buffered saline containing $0.5 \%$ bovine serum albumin. The specific activities of ${ }^{125}$ I-girentuximab and ${ }^{125}$ I-girentuximabIRDye $800 \mathrm{CW}$ were 4.0 and $7.8 \mathrm{MBq} / \mu \mathrm{g}$, respectively. In all experiments, a mAb dose of $5 \mu \mathrm{g}$ was used.
The radiochemical purity of the radiolabeled mAb preparations was determined by instant thin-layer chromatography with silica gel strips (Biodex Medical Systems) and 0.1 M citrate buffer ( $\mathrm{pH}$ 5.0) as the mobile phase. The labeling efficiency exceeded $90 \%$ for all antibody preparations. After purification by gel filtration on a PD10 column, the radiochemical purity of all preparations used in the study exceeded $95 \%$. The immunoreactive fractions of all radiolabeled girentuximab preparations, as determined with freshly trypsinized SK-RC-52 ccRCC cells essentially as described by Lindmo et al. (17), were greater than or equal to $79 \%$ for ${ }^{125}$ I-girentuximab and greater than or equal to $67 \%$ for ${ }^{125}$ I-girentuximab-IRDye $800 \mathrm{CW}$.

\section{Optical Imaging and Micro-SPECT Imaging}

Images were recorded at $1 \mathrm{~h}, 1 \mathrm{~d}$, and $3 \mathrm{~d}$ after intravenous $\mathrm{mAb}$ injection. Optical images were acquired with an IVIS Lumina imaging system (recording time, 1-3 min; binning factor, 4; emission filter, indocyanine green; field of view, 12.5; excitation filter, $745 \mathrm{~nm}$ ) (Caliper Life Sciences). Mice were placed in the prone position in the scanner, and their body temperature was maintained at $37^{\circ} \mathrm{C}$ with a heated imaging stage. All animals were gas-anesthetized with a mixture of oxygen, $\mathrm{N}_{2} \mathrm{O}$, and isoflurane. Micro-SPECT images were acquired with a USPECT II micro-SPECT scanner (MILabs) by use of the GP-RM 1.0-mm collimator tube with 75 pinholes (18). SPECT images were reconstructed by ordered-subset maximization expectation (6 iterations; 16 subsets; voxel size, $0.1875 \mathrm{~mm}$ ) with U-SPECT-Rec software (MILabs).

\section{Study Design}

Changes in girentuximab biodistribution as a result of the conjugation of the mAb to IRDye $800 \mathrm{CW}$ were assessed with 2 groups of 5 mice. Mice with a subcutaneous SK-RC-52 tumor were injected with either ${ }^{125}$ I-labeled girentuximab-IRDye800CW or unconjugated ${ }^{125}$ I-labeled girentuximab. Both groups were studied with micro-SPECT imaging, and the group receiving ${ }^{125} \mathrm{I}$-girentuximab-IRDye $800 \mathrm{CW}$ was also studied with optical imaging. Biodistribution studies were performed for both groups.

For determination of whether tumor targeting of the girentuximab constructs was CAIX antigen-mediated, 2 groups of 6 mice with a subcutaneous CAIX-positive tumor (SK-RC-52) in the left flank and a subcutaneous CAIX-negative tumor (SK-RC-59) in the right flank were used. One group of mice received ${ }^{125}$ I-girentuximab-IRDye $800 \mathrm{CW}$, and the other group received nonspecific ${ }^{125}$ I-labeled MOPC21-IRDye $800 \mathrm{CW}$. Both groups were studied with optical imaging as well as micro-SPECT imaging, and biodistribution studies were performed.

The specificity of girentuximab targeting to CAIX-expressing ccRCC tumors was studied with an additional group of 5 SK-RC-52-bearing mice. These mice were coinjected with ${ }^{125}$ I-girentuximab-IRDye $800 \mathrm{CW}$ and an excess $(500 \mu \mathrm{g})$ of unlabeled girentuximab. Again, optical imaging as well as micro-SPECT imaging and biodistribution studies were performed.

For assessment of the feasibility of image-guided surgery for ccRCC tumors, 5 mice with subcutaneous CAIX-expressing tumors were injected intravenously with ${ }^{125}$ I-girentuximab-IRDye $800 \mathrm{CW}$. Tumors were visualized with fluorescence imaging at $3 \mathrm{~d}$ after injection. Afterward, the tumors were resected, and imaging of the mice was performed again to evaluate whether radical tumor resection was achieved.

\section{Biodistribution Studies}

After the last images were acquired ( $3 \mathrm{~d}$ after injection), mice were euthanized, and the biodistribution of the radiolabel was determined. Tumors and samples of normal tissues (blood, muscle, lung, spleen, kidney, liver, small intestine, and stomach) were dissected and weighed, and counts were obtained with a $\gamma$ counter (1480 Wizard 3"; PerkinElmer). The biodistribution results are reported as the percentage injected dose (\%ID) per gram of tissue (\%ID/g). The thyroid was also dissected, and counts were obtained; the results for this organ are expressed as the 


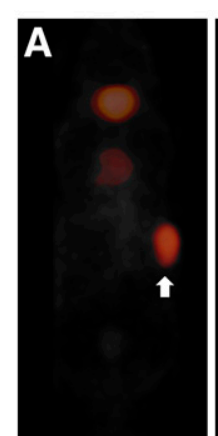

Day 1

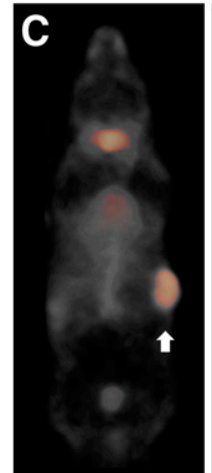

Day 1

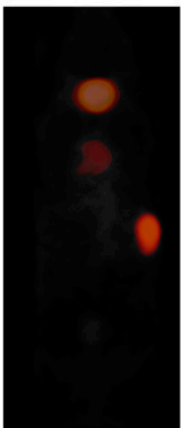

Day 3

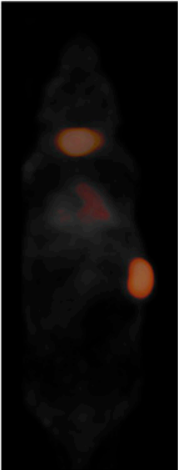

Day 3

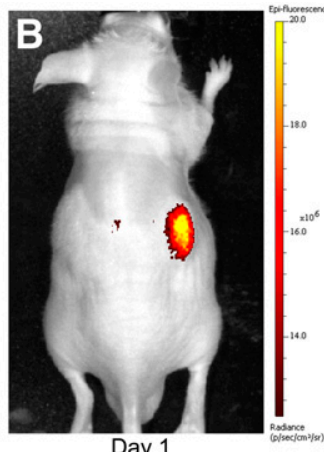

Day 1

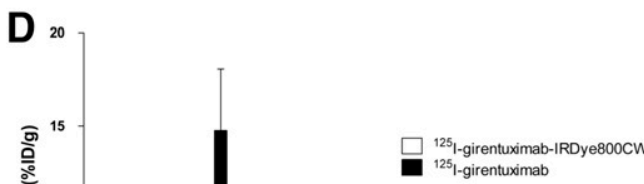

FIGURE 1. (A) Micro-SPECT images of mouse bearing SK-RC-52 tumor on right flank (arrow) at 1 and $3 \mathrm{~d}$ after injection of ${ }^{125}$ I-girentuximab-IRDye800CW. In addition to tumor uptake, minimal uptake in heart and thyroid was observed. (B) Optical images of same mouse at 1 and $3 \mathrm{~d}$ after injection (same image settings were used for days 1 and 3). Some reflectance-induced image artifacts on backs of mice were observed. (C) Micro-SPECT images of mouse bearing SK-RC-52 tumor on right flank (arrow) at 1 and $3 \mathrm{~d}$ after injection of ${ }^{125} \mathrm{l}$-girentuximab. (D) Biodistribution of ${ }^{125} \mathrm{I}$-girentuximab-IRDye800CW and ${ }^{125} \mathrm{I}$-girentuximab in mice with subcutaneous SK-RC-52 tumor at $3 \mathrm{~d}$ after injection. Values are expressed as mean $\pm \mathrm{SD}$. T/B ratio $=$ tumor-to-blood ratio.

\%ID. Counts for injection standards were obtained simultaneously to correct for radioactive decay.

\section{Statistical Analysis}

Statistical analysis was performed with $t$ tests. Differences were considered significant at a $P$ value of less than 0.05 (2-sided). All values are expressed as mean $\pm \mathrm{SD}$.
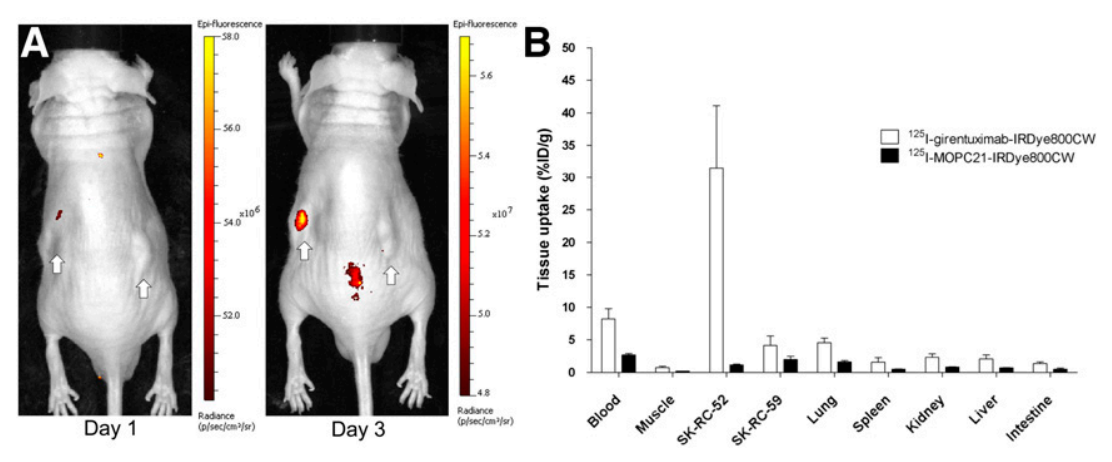

FIGURE 2. (A) Optical images of mouse bearing CAIX-positive SK-RC-52 tumor on left flank and CAIX-negative SK-RC-59 tumor on right flank (arrows) at 1 and $3 \mathrm{~d}$ after injection of 125 I-girentuximabIRDye800CW (same image settings were used for days 1 and 3). Some reflectance-induced image artifacts on backs of mice were observed. (B) Biodistribution at $3 \mathrm{~d}$ after injection of ${ }^{125}$ I-girentuximabIRDye800CW or ${ }^{125}$ I-MOPC21-IRDye800CW in mice with subcutaneous SK-RC-52 tumor and subcutaneous SK-RC-59 tumor. Values are expressed as mean \pm SD.

\section{RESULTS}

Optical images of mice bearing a subcutaneous CAIX-positive SK-RC-52 tumor and injected with ${ }^{125}$ I-girentuximab-IRDye $800 \mathrm{CW}$ showed targeting of the tumor by the IRDye800CW-conjugated antibody preparation at 1 and $3 \mathrm{~d}$ after injection (Fig. 1B). No tumor uptake was detected at $1 \mathrm{~h}$ after injection (data not shown). Micro-SPECT images of the same animals confirmed the accumulation of ${ }^{125}$ I-girentuximab-IRDye $800 \mathrm{CW}$ in the CAIX-expressing tumor (Fig. 1A). The biodistribution of the radiolabel in these mice is summarized in Figure 1D. The data (SKRC-52 uptake, $6.9 \pm 1.1 \% \mathrm{ID} / \mathrm{g}$; mean tumor weight, $0.09 \pm 0.03 \mathrm{~g}$ ) confirmed the preferential uptake of ${ }^{125}$ I-girentuximab-IRDye $800 \mathrm{CW}$ in the tumor, as visualized on the optical images and the micro-SPECT images.

Micro-SPECT images of SK-RC-52 tumor-bearing mice injected with ${ }^{125}$ I-girentuximab showed tumor uptake at 1 and $3 \mathrm{~d}$ after injection (Fig. 1C). No tumor uptake was detected at $1 \mathrm{~h}$ after injection (data not shown). The biodistribution data for ${ }^{125} \mathrm{I}-$ girentuximab confirmed the preferential tumor accumulation observed on the microSPECT images $(14.8 \pm 3.3 \% \mathrm{ID} / \mathrm{g}$; mean tumor weight, $0.09 \pm 0.06 \mathrm{~g}$ ) (Fig. 1D).

At 3 days after injection, the levels of ${ }^{125}$ I-girentuximab-IRDye $800 \mathrm{CW}$ in blood were significantly lower than those of ${ }^{125}$ I-girentuximab $(2.7 \pm 0.35$ vs. $7.1 \pm$ $1.3 \% \mathrm{ID} / \mathrm{g}$, respectively; $P=0.001$ ), suggesting faster clearance of the IRDye $800 \mathrm{CW}$ conjugated antibody preparation from the blood. However, tumor-to-blood ratios did not differ significantly between the IRDye $800 \mathrm{CW}$-conjugated antibody and the unconjugated antibody $(2.6 \pm 0.34$ vs. $2.1 \pm 0.34 \% \mathrm{ID} / \mathrm{g}$, respectively; $P=$ $0.053)$.

In the second experiment, we investigated the specificity of the CAIXmediated targeting of girentuximab by administering ${ }^{125}$ I-girentuximab-IRDye800CW or ${ }^{125}$ I-MOPC21-IRDye800CW to mice with both a CAIX-positive tumor and a CAIXnegative tumor. Optical images and biodistribution data are shown in Figure 2. At 3 days after injection, the uptake of ${ }^{125}$ I-girentuximabIRDye800CW in the CAIX-positive tumor was high $(31.5 \pm 9.6 \% \mathrm{ID} / \mathrm{g}$; tumor-to-blood ratio, $3.2 \pm 2.0$; mean tumor weight, $0.02 \pm 0.01 \mathrm{~g}$ ), whereas low uptake was observed in the CAIXnegative tumor $(4.1 \pm 1.5 \% \mathrm{ID} / \mathrm{g}$; tumor-toblood ratio, $0.4 \pm 0.2$; mean tumor weight, $0.07 \pm 0.11 \mathrm{~g}$ ). Both tumor uptake and tumorto-blood ratios were significantly different between CAIX-positive and CAIX-negative tumors $(P=0.003$ and $P=0.018$, respectively). No tumor uptake was detected at $1 \mathrm{~h}$ after injection (data not shown). Mice injected 


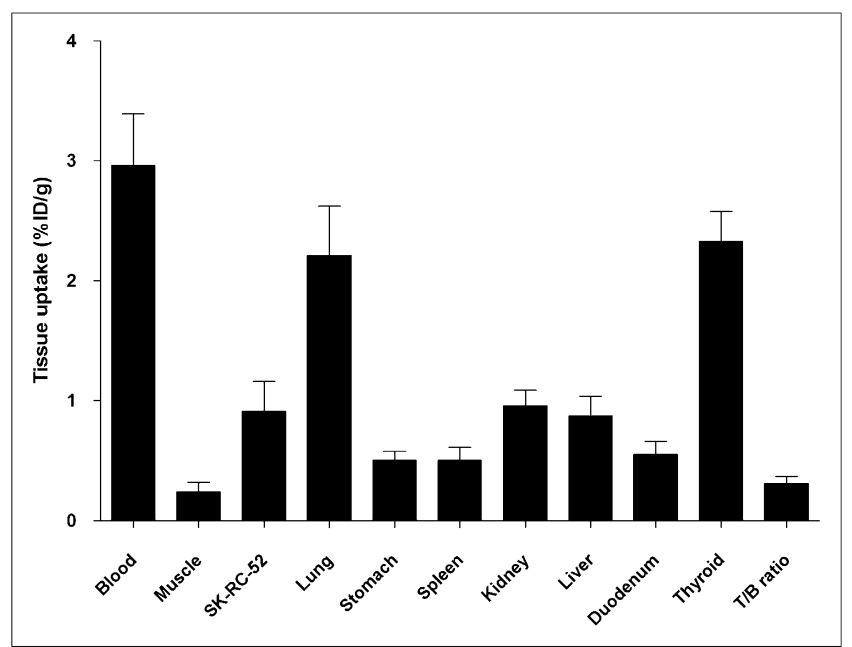

FIGURE 3. Ex vivo biodistribution of ${ }^{125}$-girentuximab-IRDye $800 \mathrm{CW}$ and excess unlabeled girentuximab. T/B ratio $=$ tumor-to-blood ratio.

with the nonspecific control conjugate ${ }^{125}$ I-MOPC21-IRDye800CW showed significantly lower tumor uptake (for CAIX-positive SK-RC$52,1.2 \pm 0.1 \% \mathrm{ID} / \mathrm{g}$; tumor-to-blood ratio, $0.4 \pm 0.1$; mean tumor weight, $0.03 \pm 0.01 \mathrm{~g}$; for CAIX-negative SK-RC-59, $2.0 \pm 0.5 \% \mathrm{ID} /$ $\mathrm{g}$; tumor-to-blood ratio, $0.5 \pm 0.4$; mean tumor weight, $0.01 \pm 0.006 \mathrm{~g})$ than mice injected with ${ }^{125}$ I-girentuximab-IRDye $800 \mathrm{CW}(P=0.002$ and $P=0.027$, respectively).

In the third experiment, the nonspecific uptake of girentuximabIRDye $800 \mathrm{CW}$ in the tumors was assessed by injecting a group of 3 mice bearing SK-RC-52 tumors with ${ }^{125}$ I-girentuximabIRDye $800 \mathrm{CW}$ and an excess of unlabeled girentuximab. MicroSPECT images acquired at $1 \mathrm{~h}, 1 \mathrm{~d}$, and $3 \mathrm{~d}$ after injection did not show any uptake of the radiolabeled $\mathrm{mAb}$ in the CAIX-expressing tumors (data not shown). Biodistribution studies confirmed that the nonspecific uptake of the IRDye800CW-conjugated antibody in these tumors was very low $(0.91 \pm 0.25 \% \mathrm{ID} / \mathrm{g}$; tumor-to-blood ratio, $0.31 \pm 0.06$; mean tumor weight, $0.13 \pm 0.08$ g) (Fig. 3).

In the fourth experiment, 5 mice with subcutaneous CAIXexpressing tumors received ${ }^{125}$ I-girentuximab-IRDye $800 \mathrm{CW}$ intravenously, and tumors were visualized with fluorescence im-

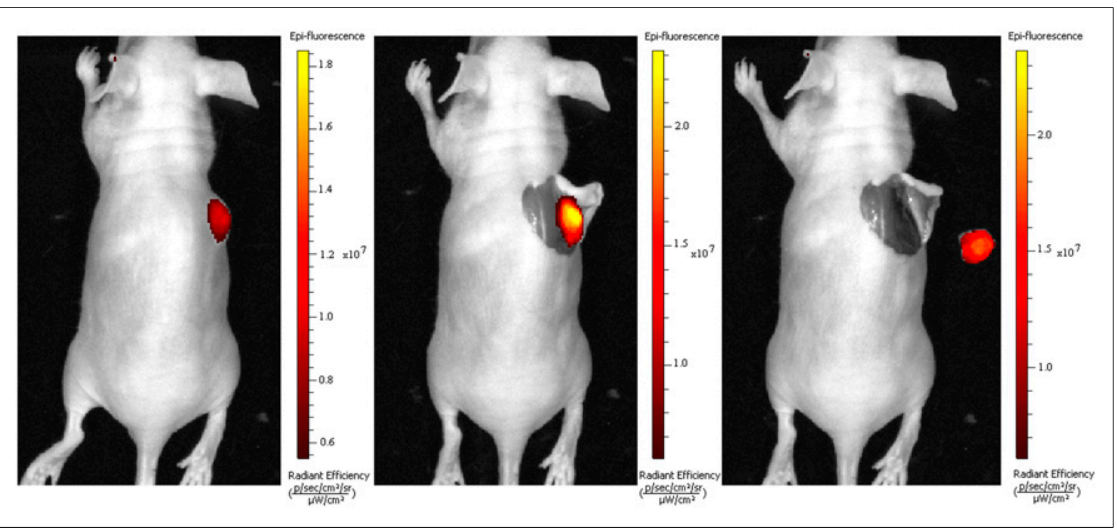

FIGURE 4. (Left) Preoperative fluorescence image of mouse with subcutaneous growing ccRCC tumor lesion at $3 \mathrm{~d}$ after injection with ${ }^{125}$-girentuximab-IRDye800CW. (Middle) Tumor lesion was subsequently removed by fluorescence image-guided surgery. (Right) After resection, no residual tumor was detected by fluorescence imaging or macroscopically. aging at $3 \mathrm{~d}$ after injection. Subsequently, the tumors were resected, and imaging of the mice was performed again to evaluate whether radical tumor resection was achieved. After resection, no residual tumor was detected by fluorescence imaging or by macroscopic examination (Fig. 4).

Taken together, these results show that ${ }^{125}$ I-girentuximabIRDye $800 \mathrm{CW}$ can target CAIX-expressing tumors with high specificity and that this targeting can be assessed by micro-SPECT and NIR fluorescence imaging. Furthermore, these results demonstrate the feasibility of targeted fluorescence image-guided surgery for ccRCC tumors.

\section{DISCUSSION}

In the present study, girentuximab-IRDye $800 \mathrm{CW}$ was characterized in a subcutaneous ccRCC xenograft model. Ex vivo biodistribution studies demonstrated high-level and specific targeting and high tumor-to-blood ratios for girentuximab-IRDye800CW in CAIX-expressing tumors. Compared with girentuximab, the IRDye800CW-conjugated antibody preparation showed lower tumor uptake at $3 \mathrm{~d}$ after injection (Fig. 1D), most likely because of faster clearance of the IRDye800CW-conjugated antibody from the circulation (8).

The preferential accumulation of dually labeled girentuximab in CAIX-expressing tumors was confirmed with both optical imaging and micro-SPECT imaging. Both imaging modalities showed that the radiolabeled girentuximab-IRDye $800 \mathrm{CW}$ conjugate accumulated specifically in CAIX-positive tumors, whereas nonspecific mAb MOPC21-IRDye800CW conjugate did not. Moreover, girentuximabIRDye800CW accumulated in CAIX-positive tumors but not in CAIX-negative tumors. These results clearly demonstrated that the accumulation of the girentuximab-IRDye $800 \mathrm{CW}$ conjugate was antigen-mediated. The tracer uptake appeared to be higher in Figure 1B than in Figure 2A because of attenuation of the fluorescence signal in tissues.

The feasibility of using girentuximab-IRDye $800 \mathrm{CW}$ in optical imaging and image-guided surgery for CAIX-expressing tumors suggests a potential role for this tracer in the intraoperative detection of positive surgical margins or tumor-infiltrated lymph nodes in patients with ccRCC tumors.

The results of the present study are in line with the results of other preclinical and clinical studies of dual-label imaging. Terwisscha van Scheltinga et al. (19) reported specific and sensitive detection of tumor lesions in vivo when they used fluorescence-labeled antibodies targeting vascular endothelial growth factor or human epidermal growth factor 2 in a breast cancer xenograft mouse model. The animals in that study received a second injection with the same antibodies labeled with a positron-emitting radionuclide, ${ }^{89} \mathrm{Zr}$, to enable a comparison between fluorescence imaging and PET imaging. The addition of a radioactive tracer seems to be indispensible for intraoperative tumor detection because of the limited depth of tissue penetration of the optical signal. However, whether the coinjection of both an optical tracer and a radiotracer will produce the same results as the ad- 
ministration of a dually labeled tracer has not yet been determined. With coinjection, the biodistribution of the optical tracer could differ from that of the radiolabeled tracer; in contrast, with a dually labeled tracer, both signals originate from the same molecule. Nontargeted dually labeled tracers were applied for sentinel lymph node biopsy and resection after intratumoral injection of nanocolloid in a clinical setting (20). The results clearly demonstrated improved lymph node detection, especially when positive nodes were located in lymph node-rich regions, as in prostate cancer or head and neck cancer $(21,22)$. A study with patients who had ovarian cancer had already shown the feasibility and potential of this approach for intraoperative fluorescence imaging in a clinical setting (23). Currently, a clinical trial of the use of fluorescence-labeled bevacizumab to enable image-guided surgery in patients with breast cancer is under way (NCT01508572). These preclinical and clinical studies demonstrate the feasibility of dual-modality imaging for image-guided surgery.

Although the present study demonstrated that the optical imaging of CAIX-expressing tumors is feasible, there are some limitations for the direct clinical translation of targeted fluorescence imaging in patients with ccRCC tumors.

In the present study, we used ${ }^{125}$ I for small-animal imaging and ex vivo biodistribution studies. Because of its long half-life $(59.4 \mathrm{~d})$ and less favorable $\gamma$ characteristics $(\gamma$ energy $=35$ $\mathrm{keV}),{ }^{125} \mathrm{I}$ is not suitable for SPECT imaging in the clinical setting. Labeling of girentuximab-IRDye $800 \mathrm{CW}$ with ${ }^{124} \mathrm{I}$ or with the residualizing radiometal ${ }^{111}$ In by use of the chelator DTPA could enable preoperative molecular imaging in patients with ccRCC tumors and thus enable both pre- and perioperative imaging of ccRCC tumors (24-27). Recently, Divgi et al. (26) assessed the accuracy of ${ }^{124}$ I-girentuximab PET/CT in a large cohort of patients with a primary renal mass scheduled for surgery. The authors found high sensitivity and specificity of ${ }^{124}$ I-girentuximab PET/CT for detecting ccRCC lesions ( $86.2 \%$ and $85.9 \%$, respectively); these results were concordant with prior experience with this tracer and suggest that this imaging modality could improve patient care in the preoperative setting (24).

A limitation of girentuximab-based imaging is that targeting depends on CAIX expression in tumor lesions. However, approximately $80 \%$ of malignant renal lesions are of the clear cell subtype, express CAIX, and therefore potentially can be imaged with girentuximab-IRDye800CW.

The results of the present study endorse the tumor-specific targeting of girentuximab and indicate that a new application for girentuximab-based imaging-namely, the intraoperative detection of ccRCC tumors, positive resection margins, and metastases with fluorescence imaging-may be feasible. This approach may lead to better cancer management. Further studies in which orthotopic or metastatic models are used to evaluate the sensitivity and potential added value of this approach are warranted.

\section{CONCLUSION}

CAIX-expressing subcutaneous ccRCC xenografts were visualized by optical imaging with ${ }^{125}$ I-girentuximab-IRDye $800 \mathrm{CW}$, with good concordance between fluorescence images and micro-SPECT images. The accumulation of ${ }^{125}$ I-girentuximabIRDye $800 \mathrm{CW}$ in ccRCC tumors was high and specific. The use of girentuximab-IRDye $800 \mathrm{CW}$ for the intraoperative detection of CAIX-expressing tumor lesions and the assessment of residual tumor in resection margins or metastatic lesions in patients with ccRCC tumors may be feasible. Clinical studies are warranted to assess the safety, feasibility, and validity of this fluorescence imaging approach for patients with ccRCC tumors.

\section{DISCLOSURE}

The costs of publication of this article were defrayed in part by the payment of page charges. Therefore, and solely to indicate this fact, this article is hereby marked "advertisement" in accordance with 18 USC section 1734. Egbert Oosterwijk, Peter F.A. Mulders, Wim J.G. Oyen, and Otto C. Boerman served or now serve on an advisory board for Wilex AG, Munich, Germany. No other potential conflict of interest relevant to this article was reported.

\section{REFERENCES}

1. Chow WH, Dong LM, Devesa SS. Epidemiology and risk factors for kidney cancer. Nat Rev Urol. 2010;7:245-257.

2. Patard JJ. Incidental renal tumours. Curr Opin Urol. 2009;19:454-458.

3. Ljungberg B, Cowan NC, Hanbury DC, et al. EAU guidelines on renal cell carcinoma: the 2010 update. Eur Urol. 2010;58:398-406.

4. Weight CJ, Lythgoe C, Unnikrishnan R, Lane BR, Campbell SC, Fergany AF. Partial nephrectomy does not compromise survival in patients with pathologic upstaging to $\mathrm{pT} 2 / \mathrm{pT} 3$ or high-grade renal tumors compared with radical nephrectomy. Urology. 2011;77:1142-1146.

5. Sutherland SE, Resnick MI, MaClennan GT, Goldman HB. Does the size of the surgical margin in partial nephrectomy for renal cell cancer really matter? J Urol. 2002; 167:61-64.

6. Kovar JL, Simpson MA, Schutz-Geschwender A, Olive DM. A systematic approach to the development of fluorescent contrast agents for optical imaging of mouse cancer models. Anal Biochem. 2007;367:1-12.

7. Cohen R, Stammes MA, de Roos IH, Stigter-van Walsum M, Visser GW, van Dongen GA. Inert coupling of IRDye800CW to monoclonal antibodies for clinical optical imaging of tumor targets. EJNMMI Res. 2011;1:31.

8. Cohen R, Vugts DJ, Stigter-van Walsum M, Visser GW, van Dongen GA. Inert coupling of IRDye $800 \mathrm{CW}$ and zirconium- 89 to monoclonal antibodies for single- or dual-mode fluorescence and PET imaging. Nat Protoc. 2013;8: 1010-1018.

9. Stillebroer AB, Mulders PF, Boerman OC, Oyen WJ, Oosterwijk E. Carbonic anhydrase IX in renal cell carcinoma: implications for prognosis, diagnosis, and therapy. Eur Urol. 2010;58:75-83.

10. Muselaers S, Mulders P, Oosterwijk E, Oyen W, Boerman O. Molecular imaging and carbonic anhydrase IX-targeted radioimmunotherapy in clear cell renal cell carcinoma. Immunotherapy. 2013;5:489-495.

11. Ebert T, Bander NH, Finstad CL, Ramsawak RD, Old LJ. Establishment and characterization of human renal cancer and normal kidney cell lines. Cancer Res. 1990;50:5531-5536.

12. Brouwers A, Verel I, Van Eerd J, et al. PET radioimmunoscintigraphy of renal cell cancer using ${ }^{89} \mathrm{Zr}$-labeled cG250 monoclonal antibody in nude rats. Cancer Biother Radiopharm. 2004;19:155-163.

13. Oosterwijk E, Ruiter DJ, Hoedemaeker PJ, et al. Monoclonal antibody G250 recognizes a determinant present in renal-cell carcinoma and absent from normal kidney. Int J Cancer. 1986;38:489-494.

14. Steffens MG, Boerman OC, Oosterwijk-Wakka JC, et al. Targeting of renal cell carcinoma with iodine-131-labeled chimeric monoclonal antibody G250. J Clin Oncol. 1997;15:1529-1537.

15. Leibovich BC, Sheinin Y, Lohse CM, et al. Carbonic anhydrase IX is not an independent predictor of outcome for patients with clear cell renal cell carcinoma. J Clin Oncol. 2007;25:4757-4764.

16. Thiry A, Dogne JM, Masereel B, Supuran CT. Targeting tumor-associated carbonic anhydrase IX in cancer therapy. Trends Pharmacol Sci. 2006;27: 566-573.

17. Lindmo T, Boven E, Cuttitta F, Fedorko J, Bunn PA Jr. Determination of the immunoreactive fraction of radiolabeled monoclonal antibodies by linear extrapolation to binding at infinite antigen excess. J Immunol Methods. 1984;72:77-89. 
18. van der Have F, Vastenhouw B, Ramakers RM, et al. U-SPECT-II: an ultrahigh-resolution device for molecular small-animal imaging. J Nucl Med. 2009;50:599-605.

19. Terwisscha van Scheltinga AG, van Dam GM, Nagengast WB, et al. Intraoperative near-infrared fluorescence tumor imaging with vascular endothelial growth factor and human epidermal growth factor receptor 2 targeting antibodies. $\mathrm{J}$ Nucl Med. 2011;52:1778-1785.

20. van der Poel HG, Buckle T, Brouwer OR, Valdes Olmos RA, van Leeuwen FW. Intraoperative laparoscopic fluorescence guidance to the sentinel lymph node in prostate cancer patients: clinical proof of concept of an integrated functional imaging approach using a multimodal tracer. Eur Urol. 2011;60:826-833.

21. van den Berg NS, Valdes-Olmos RA, van der Poel HG, van Leeuwen FW. Sentinel lymph node biopsy for prostate cancer: a hybrid approach. $J$ Nucl Med. 2013;54:493-496.

22. Brouwer OR, Buckle T, Vermeeren L, et al. Comparing the hybrid fluorescentradioactive tracer indocyanine green- ${ }^{99 \mathrm{~m}} \mathrm{Tc}$-nanocolloid with ${ }^{99 \mathrm{~m}} \mathrm{Tc}$-nanocolloid for sentinel node identification: a validation study using lymphoscintigraphy and SPECT/CT. J Nucl Med. 2012;53:1034-1040.
23. van Dam GM, Themelis G, Crane LM, et al. Intraoperative tumor-specific fluorescence imaging in ovarian cancer by folate receptor-alpha targeting: first inhuman results. Nat Med. 2011;17:1315-1319.

24. Divgi CR, Pandit-Taskar N, Jungbluth AA, et al. Preoperative characterisation of clear-cell renal carcinoma using iodine-124-labelled antibody chimeric G250 $\left({ }^{124} \mathrm{I}-\mathrm{cG} 250\right)$ and PET in patients with renal masses: a phase I trial. Lancet Oncol. 2007;8:304-310.

25. Stillebroer AB, Boerman OC, Desar IM, et al. Phase 1 radioimmunotherapy study with lutetium 177-labeled anti-carbonic anhydrase IX monoclonal antibody girentuximab in patients with advanced renal cell carcinoma. Eur Urol. 2013;64:478-485.

26. Divgi CR, Uzzo RG, Gatsonis C, et al. Positron emission tomography/computed tomography identification of clear cell renal cell carcinoma: results from the REDECT trial. J Clin Oncol. 2013;31:187-194.

27. Muselaers CH, Boerman OC, Oosterwijk E, Langenhuijsen JF, Oyen WJ, Mulders PF. Indium-111-labeled girentuximab immunoSPECT as a diagnostic tool in clear cell renal cell carcinoma. Eur Urol. 2013;63:11011106 